\title{
Mysterious cosmic radio blasts traced to surprising source
}

\section{Repeating bursts come from a faint, distant dwarf galaxy.}

\section{Alexandra Witze}

04 January 2017

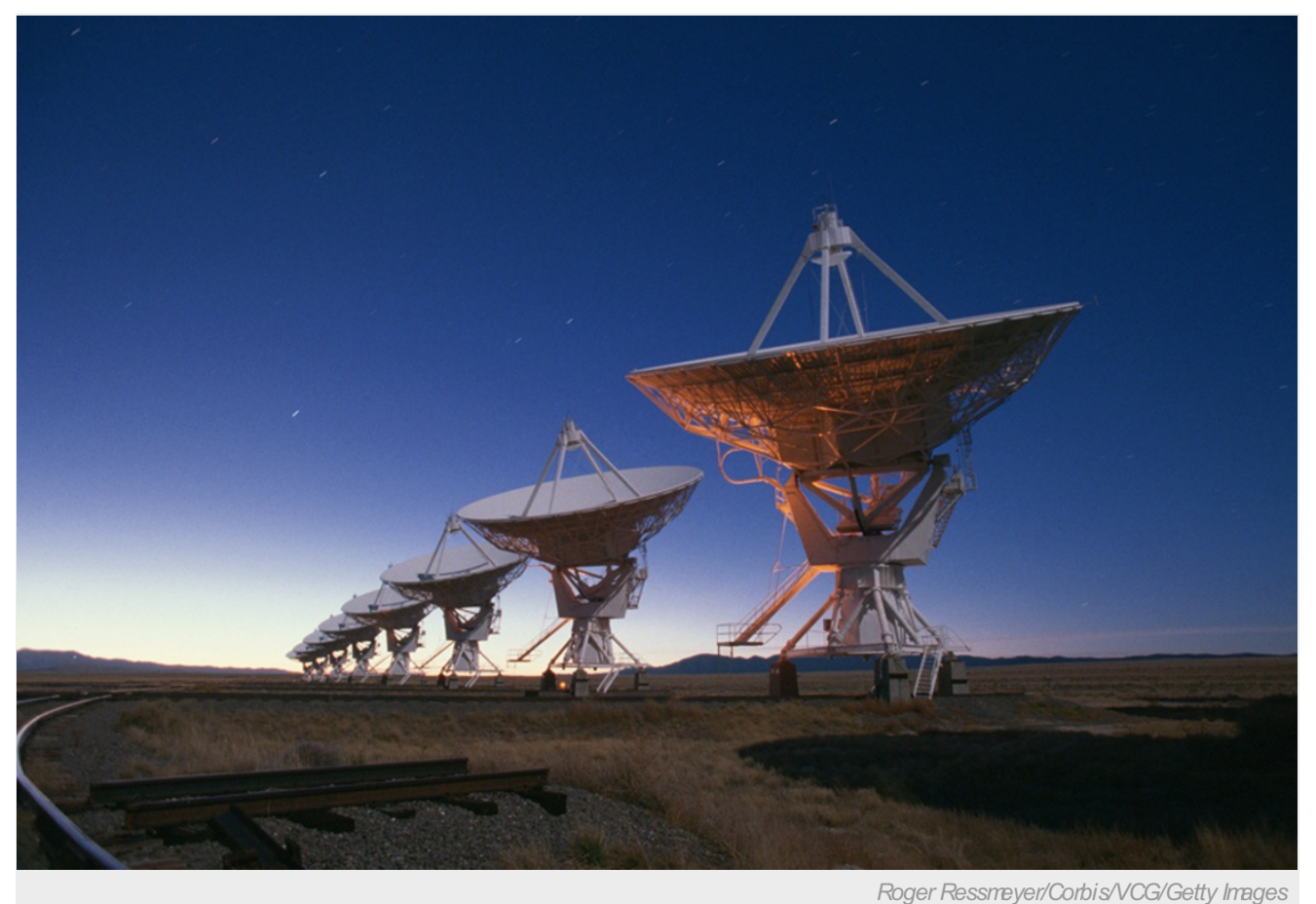

Radio telescopes at New Mexico's Very Large Array are helping to solve the mystery of fast radio bursts.

Astronomers have pinpointed the location of an enigmatic celestial object that spits out brief, but powerful, blasts of radio waves.

Surprisingly, the source of these intermittent signals lies not in a bright galaxy but in a small, dim one, some 2.5 billion light-years from Earth.

The discovery begins to lift the curtain on the mystery of fast radio bursts, which have puzzled astronomers since they first described the signals in $2007^{1}$. "This detection has really broken open the gates of a new realm of science and discovery," says Sarah BurkeSpolaor, an astronomer at the National Radio Astronomy Observatory in Socorro, New Mexico, and West Virginia University in Morgantown. She spoke in Grapevine, Texas, at a meeting of the American Astronomical Society.

Fast radio bursts appear to come from beyond the Milky Way and crop up seemingly at random across the sky. Although they last just milliseconds, the radio blasts can emit as much power as 500 million Suns.

The bursts were first spotted by the Parkes radio telescope in New South Wales, Australia, and fewer than 20 have been found so far. Most were discovered in wide-field searches that cannot pinpoint exactly where they come from — which makes it harder for astronomers to winnow down possible explanations for what causes them.

\section{A puny host}

The latest work, published on 4 January in Nature ${ }^{2}$, is the sharpest look yet at the home of a fast radio burst known as FRB 121102. Located in the constellation Auriga, the intermittent signal was first detected on 2 November 2012. Since then, it has flared up several times, making it the only fast radio burst known to repeat ${ }^{3}$.

A team led by Shami Chatterjee, an astronomer at Cornell University in Ithaca, New York, began with the 305-metre-wide Arecibo radio telescope in Puerto Rico. Its sensitivity allowed the scientists to detect multiple bursts from FRB 121102. The team then used two sets of radio telescopes — the Karl G. Jansky Very Large Array in New Mexico, and the European VLBI Network across Europe — to narrow down the location of FRB 121102 even further ${ }^{4}$. 
The bursts originate from a dwarf galaxy that emits faint radiation in both radio and visual wavelengths. Follow-up observations with the Gemini North telescope, on Mauna Kea, Hawaii, showed that it is less than one-tenth the size and has less than one-thousandth the mass of the Milky Way ${ }^{5}$.

"The host galaxy is puny," says team member Shriharsh Tendulkar, an astronomer at McGill University in Montreal, Canada. "That's weird." With fewer stars than many galaxies, dwarf galaxies would seem to have less of a chance of hosting whatever creates fast radio bursts. That would include neutron stars, one of the leading candidates for the source of fast radio bursts.

But much more work is needed to pin down the physical mechanism of what causes these mysterious bursts, says Chatterjee. For now, FRB 121102 is just one example.

That need could be filled later this year when a new radio telescope comes online in British Columbia, Canada, dedicated to hunting fast radio bursts.

Nature | doi:10.1038/nature.2016.21235

\section{References}

1. Lorimer, D. R., Bailes, M. McLaughlin, M. A., Narkevic, D. J., \& Crawford, F. Science 318, 777-780 (2007).

2. Chatterjee, S. et al. Nature http://dx.doi.org/10.1038/nature20797 (2017).

3. Spitler, L. G. et al. Nature 531, 202-205 (2016).

4. Marcote, B. et al. Astrophys. J. Lett. 834, L8 (2017)

5. Tendulkar, S. P. et al. Astrophys. J. Lett. 834, L7 (2017). 